\section{Nível de atividade física no lazer em usuários do sistema único de saúde}

\section{Level of leisure physical activity in users of the Brazilian public health system}

\author{
Jamile Sanches Codogno ${ }^{1}$ \\ Bruna Camilo Turi ${ }^{1,3}$ \\ Rômulo Araújo Fernandes ${ }^{2}$ \\ Henrique Luiz Monteiro ${ }^{4}$
}

\section{Resumo}

Embora se investigue a atividade física no lazer na população em geral, muito pouco se sabe sobre está variável entre pacientes do Sistema Único de Saúde. Assim, o objetivo deste estudo foi analisar o nível de atividade física no lazer de pacientes atendidos na rede publica de saúde de Bauru-SP, bem como, identificar alguns de seus determinantes e sua associação com alguns agravos à saúde. A amostra foi composta por 963 pacientes (707 mulheres e 256 homens) selecionados aleatoriamente em cinco unidades básicas de saúde. As atividades físicas no lazer foram avaliadas pelo questionário de Baecke e indivíduos que reportaram $\geq 180$ ou $\geq 240$ minutos por semana de atividades físicas foram considerados ativos e muito ativos. Obesidade geral e obesidade central foram identificadas por valores de índice de massa corporal e circunferência de cintura, respectivamente. As doenças de maior ocorrência foram agrupadas segundo o CID-10. Houve baixa prática suficiente de atividade física no lazer $(14,8 \%)$, mas elevada taxa de obesidade abdominal $(70,1 \%)$ e doenças do aparelho osteomuscular e do tecido conjuntivo $(72,1 \%)$. Indivíduos fisicamente ativos no lazer apresentaram menor ocorrência de doenças do aparelho osteomuscular e do tecido conjuntivo $\left(\mathrm{OR}=0.60\left[\mathrm{OR}_{\mathrm{IC} 95 \%}: 0.37-0.98\right]\right)$. A mesma associação foi observada para o sexo feminino no que diz respeito à obesidade abdominal $(\mathrm{OR}=0.35$ $\left.\left[\mathrm{OR}_{\mathrm{IC} 95 \%}: 0.19-0.65\right]\right)$. É baixa a prática suficiente de física no lazer entre pacientes do Sistema Único de Saúde, a qual está associada à menor ocorrência de doenças do aparelho osteomuscular e do tecido conjuntivo.

\section{Palavras-chave}

Atividade motora; Atenção primária à saúde; Estudo epidemiológico; Brasil.

\begin{abstract}
Although leisure physical activity has been investigated in general population, there is absence of data in Brazilian Public Health System about this issue. Thus, the purpose of this study was to analyze in patients attended by Public Health System the prevalence of leisure physical activity, as well as, to identify its correlates and associated diseases. Sample was composed by 963 patients ( 707 female and 256 male) randomly selected at five basic healthcare units. Leisure physical activity was assessed by Baecke's questionnaire and subjects with $\geq 180$ and $\geq 240$ minutes per week were classified as sufficiently active. General and central obesity were assessed by body mass index and waist circumference, respectively. Diseases of high occurrence were classified according CID-10. There was low rate of physically active subjects in leisure time (14.8\%), but high rate of abdominal obesity (70.1\%) and orthopedic diseases (72.1\%). Physically active subjects in leisure time had lower occurrence of orthopedic diseases (OR= $\left.0.60\left[O R_{95 \% C r} \cdot 0.37-0.98\right]\right)$. The same pattern of association was observed for central obesity $(O R=$ $\left.0.35\left[O R_{95 \% \mathrm{Cr}} \cdot 0.19-0.65\right]\right)$. It is low the rate of physically active in leisure time at Brazilian Public Health System, which is associated with orthopedic diseases.
\end{abstract}

\section{Keywords}

Physical activity; Primary healthcare; Epidemiological study; Brazil.
Rev Bras Ativ Fis Saúde p. 543-551 DOI: http://dx.doi.org/10.12820/23171634.2012v17n6p543

1 Programa de Pós-Graduação em Ciências da Motricidade. Universidade Estadual Paulista, Rio Claro, SP, Brasil.

2 Departamento de Educação Física. Universidade Estadual Paulista, Presidente Prudente, SP, Brasil.

3 Programa de Pós-Graduação em Fisioterapia. Universidade Estadual Paulista, Presidente Prudente, SP, Brasil.

4 Departamento de Educação Física. Universidade Estadual Paulista, Bauru, SP Brasil. 


\section{INTRODUÇÃO}

A Constituição Federal brasileira assegura a todos o direito à saúde, que deve ser ofertado pelas três esferas do poder público, por meio da articulação e interação dentro do Sistema Único de Saúde (SUS) ${ }^{1}$. Neste contexto, as estratégias de ação da atenção básica no Brasil têm se direcionado para o desenvolvimento de políticas de promoção, proteção e recuperação de saúde, visando à elaboração de iniciativas que afetem positivamente a qualidade de vida da população através da Política Nacional de Promoção da Saúde (PNPS)².

A PNPS, através de estudos que abordam o sedentarismo como problema de saúde pública, incentiva à prática de atividades físicas bem como reconhece a importância de proporcionar à população, espaços prazerosos e adequados, com segurança, arborização e transporte público ${ }^{3}$. Nesse sentido, em virtude de outros domínios da atividade física apresentarem dificuldades em sua modificação (caso de atividades ocupacionais), a atividade física no lazer parece receber um destaque especial em campanhas de promoção da atividade física, pois, pode ser modificada ( pode-se optar por ler um livro ou realizar uma caminhada ao ar livre).

É bem documentado na literatura o efeito da prática de atividades físicas para a prevenção, tratamento de doenças ${ }^{4,5}$, e controle da obesidade ${ }^{6}$, aspecto diretamente relacionado ao surgimento de inúmeros agravos crônicos. $\mathrm{O}$ aumento na prevalência destas doenças sobrecarrega os serviços de saúde ${ }^{7}$, gerando aumento dos gastos públicos e privados. Estudo realizado em nosso meio aponta que a atividade física pode modificar beneficamente parâmetros fisiológicos e, consequentemente, contribuir para a redução dos gastos com serviços de saúde ${ }^{8}$.

Por outro lado, embora os usuários do Sistema Único de Saúde representem aproximadamente $60 \%$ da população brasileira, pouco se conhece sobre a prática de atividades físicas entre pessoas que têm no sistema público de atenção à saúde sua única alternativa. Neste caso, sobre sua associação com doenças, observa-se que nos pacientes assistidos pelo SUS quanto menor o nível de atividade física maior o número de agravos à saúde ${ }^{9}$. Tais informações são importantes para a orientação de gestores públicos na promoção e estruturação de campanhas de promoção da prática de atividades físicas nesta população. Além disso, pessoas com cinquenta anos ou mais apresentam significativo aumento na ocorrência de obesidade e doenças crônicas ${ }^{4,5,9}$, e são foco de campanhas de prevenção à tais desfechos.

Dessa forma, os objetivos do presente estudo foram: (i) analisar o nível de atividade física no lazer de pacientes atendidos pela rede publica de saúde, (ii) identificar alguns fatores associados e (iii) verificar sua associação com alguns agravos a saúde destes pacientes.

\section{MÉTODOS}

\section{Casuística}

O projeto foi aprovado pelo Comitê de Ética em Pesquisa da Faculdade de Ciências da Universidade Estadual Paulista - Campus de Bauru (n. ${ }^{\circ}$ 1046/46/01/10). A amostra foi composta por 963 adultos de ambos os sexos, atendidos nas cinco maiores Unidades Básicas de Saúde - UBS da cidade de Bauru-SP (a maior em cada uma das cinco regiões: leste, oeste, norte, sul e centro). Para o cálculo do tamanho da amostra, considerou-se uma prevalência de prática suficiente de atividade física no lazer de $16,6 \%{ }^{4}$, bem como, um erro amostral de $3 \%$, significância de $5 \%$ ( $\mathrm{z}=1,96$ por utilizar um IC95\%) e um efeito de design de 50\% (por utilizar 
amostragem por conglomerado, neste caso, as cinco UBS), a amostra total foi estimada em 886 sujeitos.

Em cada uma das cinco UBS selecionadas, após triagem inicial das agendas médicas (na qual eram computados todos os pacientes que passaram pela unidade nos últimos seis meses), os pacientes que atendiam aos critérios de inclusão (idade $\geq 50$ anos [por ser um grupo etário, no qual a ocorrência de doenças crônicas já se manifesta de maneira importante $]^{4,5,9}$ e, pelo menos, uma consulta nos últimos seis meses) foram inseridos em uma listagem de pacientes a serem sorteados. Em seguida, a partir do número do prontuário, entre 250 e 500 pacientes foram selecionados por meio de sorteio aleatório (realizado através do software estatístico Statistical Package for the Social Sciences [SPSS], versão 13.0). Os pacientes sorteados foram convidados por contato telefônico a comparecerem na UBS para realização de avaliação e aplicação de questionários, sendo que, quando o número mínimo de indivíduos não foi atendido no primeiro sorteio, outros foram realizados. Estas UBS foram selecionadas para a realização do estudo, pois, o número de prontuários cadastrados nas mesmas (114.384 prontuários) representa mais de 50\% do total de pacientes que usam exclusivamente o SUS na cidade de Bauru (60\% da população) ${ }^{10}$.

\section{Doenças, obesidade total e abdominal}

Para detectar a presença de doenças foi utilizado inquérito de morbidades referidas desenvolvido pelo Standard Health Questionnaire for Washington State e adaptado para a população brasileira por Freitas Júnior et al. ${ }^{11}$. O instrumento é constituído por questões fechadas que permitem identificar a presença ou ausência de doenças crônicas, as quais foram identificadas de acordo com a Classificação Estatística Internacional de Doenças e Problemas Relacionados à Saúde ${ }^{12}$. No presente estudo, em decorrência do grande número de doenças detectado nos prontuários, foram utilizados nas análises apenas os três grupos de doenças mais prevalentes na amostra (CIDE00-E90= doenças endócrinas, nutricionais e metabólicas; CIDI00-I99= doenças do aparelho circulatório; CIDM00-M99= doenças do aparelho osteomuscular e do tecido conjuntivo).

O Índice de Massa Corporal (IMC; em $\mathrm{kg} / \mathrm{m}^{2}$ ) foi calculado com a utilização dos valores de peso corporal e estatura, coletados segundo o protocolo de Lohman et al. $^{13}$. A presença do sobrepeso/obesidade foi diagnosticada quando o IMC apresentou valor entre 25 e $29.9 \mathrm{~kg} / \mathrm{m}^{2}$ para sobrepeso e $\geq 30 \mathrm{~kg} / \mathrm{m}^{2}$ para obesidade ${ }^{14}$. A presença da obesidade abdominal foi obtida através da avaliação da circunferência de cintura que foi coletada seguindo o protocolo proposto por Lohman et al. ${ }^{13}$. Os pontos de corte $102 \mathrm{~cm}$ para homens e $88 \mathrm{~cm}$ para mulheres foram utilizados para determinar a CC elevada ${ }^{15}$.

\section{Prática de atividades físicas}

O questionário de Baecke et al. ${ }^{16}$, é utilizado para identificar três diferentes domínios de atividade física a saber: ocupacional; esportes; lazer e locomoção. Para efeito de estudo, somente o de lazer foi considerado, particularmente porque este domínio é diretamente associado aos benefícios da atividade física relacionados à saúde. No lazer, as atividades físicas dos pacientes foram classificadas segundo a quantidade de minutos que praticavam determinados exercícios físicos/semana. Para composição dos grupos foram analisados três construtos dessa prática de atividades físicas durante horários de lazer: intensidade (baixa, moderada e vigorosa), tempo semanal de prática ( $<1 \mathrm{~h} / \mathrm{sem} ; 1-2 \mathrm{~h} / \mathrm{sem} ; 2-3 \mathrm{~h} / \mathrm{sem} ; 3-4 \mathrm{~h} / \mathrm{sem} ;>4 \mathrm{~h} / \mathrm{sem})$ e tempo prévio de engajamento ( $<1$ mês; $1-3$ meses; $4-6$ meses; $7-9$ meses; $>9$ me- 
ses). Assim, foram considerados fisicamente ativos os indivíduos que relataram ou 180 minutos $(3-4 \mathrm{~h} / \mathrm{sem})$ ou 240 minutos $(>4 \mathrm{~h} / \mathrm{sem})$ por semana de atividades físicas de intensidade moderada ou vigorosa, nos últimos quatro meses (4-6 meses) ${ }^{4,5}$.

No presente estudo, buscou-se analisar a possibilidade do período de duração da atividade física no lazer estar associado a menor ocorrência dos desfechos analisados. Embora se reconheça a limitação do delineamento transversal, buscou-se visualizar um possível comportamento de "dose resposta" entre as variáveis. Assim, os pacientes foram enquadrados em um dos grupos de atividades físicas, como segue: i) nenhuma atividade física por semana; ii) que realizam entre 180 e 239 minutos na semana e iii) com mais de $\geq 240$ minutos de atividade física na semana.

\section{Variáveis de confusão}

Algumas variáveis foram definidas como potenciais fatores: idade [estratificada em: $<65$ anos e $\geq 65$ anos], sexo (masculino e feminino) e condição econômica (CE) (alta - A e B; baixa - C, D e E). Especificamente para determinação da CE foi utilizado questionário desenvolvido pela Associação Brasileira de Empresas de Pesquisa $^{17}$, no qual a subdivisão da $\mathrm{CE}$ se dá de $\mathrm{A}$ (mais alta) a $\mathrm{E}$ (mais baixa). A amostra foi subdividida em: $\mathrm{CE}$ alta (categorias A e B) e baixa (categorias C, D e E) como adotado por Fernandes et al. $(2008)^{18}$.

\section{Análise estatística}

O teste de normalidade (Kolmogorov-Smirnov) rejeitou o enquadramento das variáveis numéricas no modelo de distribuição normal, assim, a estatística descritiva foi composta por valores de mediana (tendência central) e diferença entre o percentil 75 e percentil 25 (dispersão), bem como, o teste "U" de Mann-Whitney estabeleceu comparações quando existiam duas variáveis numéricas independentes.

Os dados categóricos foram expressos em frequências relativas e seus respectivos intervalos de confiança de 95\% (IC95\%). O teste qui-quadrado $\left(\chi^{2}\right)$ analisou a associação entre os dados categóricos (correção de Yates foi empregada em tabelas de contingência com configuração $2 \times 2$ ), bem como, a regressão logística binária (expressa em valores de odds ratio [OR] e seus respectivos intervalos de confiança de $\left.95 \%\left[\mathrm{OR}_{\mathrm{IC} 95 \%}\right]\right)$ indicou a magnitude destas associações. No modelo multivariado de regressão logística binária, foram analisadas apenas as variáveis significativamente associadas no modelo univariado e as variáveis de confusão foram inseridas simultaneamente. Vale salientar que, para responder os objetivos propostos, dependendo da análise estatística empregada, a atividade física de lazer foi tratada como variável dependente ou independente.

Todos os procedimentos acima descritos foram efetuados através do software estatístico Statistical Package for the Social Sciences - SPSS (versão 13.0) e adotou-se uma significância estatística $(P)$ inferior a $5 \%$.

\section{RESULTADOS}

Ao todo 1201 pacientes foram contatados por telefone, porém, 115 não quiseram participar e 123 faltaram no dia agendado para a avaliação. Assim, a amostra foi composta por 963 pacientes. Dos 963 pacientes, 73,4\% eram do sexo feminino (idade: $65 \pm 9$ anos [mínimo: 50 e máximo: 96]). Quanto ao perfil dos usuários, em relação ao poder aquisitivo, 83,2\% é proveniente das classes C, D e E. Observouse $66,7 \%$ de sedentarismo, $5,7 \%$ de indivíduos que praticavam $180-239 \mathrm{~min} / \mathrm{sem}$ de atividades de lazer e $9 \%$ de indivíduos com duração $\geq 240 \mathrm{~min} / \mathrm{sem}$ (Tabela 1). 
Tabela 1 - Características gerais de pacientes atendidos em unidades básicas de saúde (Bauru -SP, n=963).

\begin{tabular}{|c|c|c|c|c|}
\hline \multirow[t]{2}{*}{ Variáveis } & $\begin{array}{l}\text { Homens } \\
(n=256)\end{array}$ & $\begin{array}{l}\text { Mulheres } \\
(n=705)\end{array}$ & $\begin{array}{c}\text { Total } \\
(n=961)\end{array}$ & \multirow[t]{2}{*}{$P$} \\
\hline & Mediana (DQ) & Mediana (DQ) & Mediana (DQ) & \\
\hline Idade (anos) & $64,4(13,2)$ & $63,3(13,2)$ & $63,7(13,6)$ & 0,037 \\
\hline$M C(\mathrm{~kg})$ & $76,1(19,9)$ & $69(19,7)$ & $71,3(19,3)$ & 0,001 \\
\hline Estatura (m) & $1,66(0,09)$ & $153,7(0,08)$ & $1,56(0,11)$ & 0,001 \\
\hline IMC (kg/m²) & $27,4(5,3)$ & $29,1(7,2)$ & $28,6(7,1)$ & 0,001 \\
\hline $\mathrm{CC}(\mathrm{cm})$ & $99,4(16,8)$ & $98,7(16,6)$ & $99,1(16,4)$ & 0,114 \\
\hline \multicolumn{5}{|l|}{ Doenças (\%) } \\
\hline CIDI00-199 & 82,4 & 79,2 & 80,1 & 0,312 \\
\hline CIDE00-E90 & 50,4 & 59,3 & 56,9 & 0,017 \\
\hline CIDM00-M99 & 54,3 & 78,5 & 72,1 & 0,001 \\
\hline \multicolumn{5}{|l|}{ Adiposidade (\%) } \\
\hline Obesidade & 28,9 & 44,7 & 40 & 0,001 \\
\hline O. Abdominal & 41,8 & 80,3 & 70,1 & 0,001 \\
\hline Atividade Física & & & & $0,001^{*}$ \\
\hline Nenhuma & 56,3 & 70,4 & 66,7 & \\
\hline$<180 \mathrm{~min} / \mathrm{sem}$ & 23 & 17 & 18,6 & \\
\hline 180-239min/sem & 7,4 & 5,1 & 5,7 & \\
\hline$\geq 240 \mathrm{~min} / \mathrm{sem}$ & 13,3 & 7,5 & 9,1 & \\
\hline
\end{tabular}

* = teste qui-quadrado para associação linear; $\mathrm{DQ}=$ diferença entre o percentil 75 e o percentil 25 $C C=$ circunferência de cintura; $\mathrm{O}=$ Obesidade; $M C=$ massa corporal; $I M C=$ índice de massa corporal; CIDI00-199= doenças do aparelho circulatório; CIDEOO-E90= doenças endócrinas, nutricionais e /metabólicas; CIDM00-M99= doenças do aparelho osteomuscular e do tecido conjuntivo.

$\mathrm{Na}$ amostra total, 80,1\% apresentaram doenças do CID I00-I99 (Aparelho circulatório), 56,9\% doenças do CID E00-E90 (doenças endócrinas, nutricionais e metabólicas) e 72,1\% de doenças do CID M00-M99 (doenças do aparelho osteomuscular/tecido conjuntivo).

No que se refere à prática suficiente de atividades físicas no lazer (ao menos $180 \mathrm{~min} / \mathrm{sem}$ de atividades físicas de lazer), houve associação com o sexo masculino (mulheres: $12,6 \% 20,7 \%$ com OR= $1.81\left[\mathrm{OR}_{\mathrm{IC} 95 \%}: 1.24-2.63\right]$ ). Similarmente, pessoas de menor poder aquisitivo apresentaram menor prática de atividades físicas $(13,1 \%)$ do que as pessoas de poder aquisitivo mais elevado $(22,8 \%$ com OR= $\left.1.96\left[\mathrm{OR}_{\mathrm{IC} 95 \%}: 1.28-2.98\right]\right)$ (Tabela 2).

Tabela 2 - Associação entre prática de atividades físicas no lazer e variáveis independentes (Bauru $-\mathrm{SP}, \mathrm{n}=963$ ).

\begin{tabular}{|c|c|c|c|}
\hline \multirow{3}{*}{ Variáveis independentes } & \multicolumn{3}{|c|}{ Atividade Física no Lazer $\geq 180 \mathrm{~min} / \mathrm{sem}$} \\
\hline & \multicolumn{2}{|c|}{ Teste $\chi^{2}$} & \multirow{2}{*}{$\begin{array}{l}\text { Regressão Logística } \\
\text { OR }_{\text {Bruta }}\left(\mathrm{OR}_{\mathrm{IC95 \%}}\right)\end{array}$} \\
\hline & $\mathrm{n}(\%)$ & $\mathrm{P}$ & \\
\hline Sexo & & 0,002 & \\
\hline Masculino & $53(20,7)$ & & $1.81(1.24-2.63)$ \\
\hline Feminino & $89(12,6)$ & & 1.00 \\
\hline Idade (anos) & & 0,805 & \\
\hline$<65$ & $80(15,1)$ & & 1.00 \\
\hline$\geq 65$ & $62(14,3)$ & & $0.94(0.65-1.34)$ \\
\hline Condição econômica & & 0,002 & \\
\hline A e B (alta) & $37(22,8)$ & & $1.96(1.28-2.98)$ \\
\hline$C-E$ (baixa) & $105(13,1)$ & & 1.00 \\
\hline
\end{tabular}

Teste $\chi 2=$ teste qui-quadrado; OR Bruta= odds ratio sem ajuste por variáveis de confusão; IC95\%= intervalo de confiança de $95 \%$. 
Para elaborar o modelo de regressão logística apresentado na Tabela 3, foi testada a associação entre as variáveis dependentes e as independentes. Assim, o teste qui-quadrado indicou que houve associação significativa entre a prática de atividades física de lazer e menor ocorrência de obesidade abdominal (Nenhuma: 72,1\%; <180min/sem: 67,6\%; 180-239min/sem: 67,3\%; >240min/sem: 62,1\%; $\mathrm{P}=$ $0,035)$ e doenças do aparelho osteomuscular/tecido conjuntivo - CID M00-M99 (Nenhuma: 74,6\%; <180min/sem: 71,5\%; 180-239min/sem: 63,6\%; >240min/ sem: 59,8\%; $\mathrm{P}=0,001)$. Por outro lado, sobrepeso/obesidade $(\mathrm{P}=0,379)$, doenças do CID E00-E90 (P= 0,836) e do CID I00-I99 (P= 0,380) não foram associadas significativamente com a prática de atividades físicas.

Tabela 3 - Modelo multivariado para a associação entre prática de atividades físicas no lazer, obesidade abdominal e doenças do aparelho osteomuscular e do tecido conjuntivo (Bauru - SP, $n=963$ ).

\begin{tabular}{|c|c|c|c|c|}
\hline \multirow[b]{2}{*}{$\mathrm{AF}_{(\mathrm{min} / \mathrm{sem})}$} & \multicolumn{2}{|c|}{ CIDM00-M99 } & \multicolumn{2}{|c|}{ Obesidade abdominal } \\
\hline & $\mathrm{OR}_{\text {Bruta }}(\mathrm{IC95 \%})$ & $\mathrm{OR}_{\text {ajust }}(\mathrm{IC} 95 \%)$ & $\mathrm{OR}_{\text {Bruta }}(\mathrm{IC} 95 \%)$ & $\mathrm{OR}_{\mathrm{ajust}}(\mathrm{IC} 95 \%)$ \\
\hline Nenhuma & 1,00 & 1,00 & 1,00 & 1,00 \\
\hline$<180$ & $0,85(0,59 ; 1,23)$ & $0,99(0,66 ; 1,46)$ & $0,80(0,56 ; 1,15)$ & $0,98(0,66 ; 1,45)$ \\
\hline $180-239$ & $0,59(0,33 ; 1,06)$ & $0,71(0,39 ; 1,30)$ & $0,79(0,44 ; 1,43)$ & $1,00(0,53 ; 1,91)$ \\
\hline$\geq 240$ & $0,50(0,31 ; 0,80)$ & $0,60(0,37 ; 0,98)$ & $0,63(0,39 ; 0,99)$ & $0,83(0,50 ; 1,39)$ \\
\hline
\end{tabular}

$\mathrm{AF}=$ atividade física; ORajust= odds ratio ajustada por sexo, idade e condição econômica; IC95\%= intervalo de confiança de 95\%; CIDI00-199= doenças do aparelho circulatório; CIDM00-M99= doenças do aparelho osteomuscular e do tecido conjuntivo.

Por fim, o modelo multivariado foi elaborado apenas para as variáveis significativas nos testes estatísticos univariados (Tabela 3). Após o ajuste pelas variáveis de confusão (sexo, idade e condição econômica), a obesidade abdominal não foi mais associada significativamente à prática de atividades físicas no lazer $(\mathrm{OR}=$ $\left.0.83\left[\mathrm{OR}_{\mathrm{IC} 95 \%}: 0.50 ; 1.39\right]\right)$. Esta associação foi afetada pelo sexo, uma vez que apenas entre as mulheres estas duas variáveis foram associadas inversamente $(\mathrm{OR}=$ $\left.0.35\left[\mathrm{OR}_{\mathrm{IC} 95 \%}: 0.19 ; 0.65\right]\right)$, sem influência do poder aquisitivo e da idade. Por outro lado, independente dos ajustes efetuados, indivíduos com $\geq 240 \mathrm{~min} / \mathrm{sem}$ de atividade físicas no lazer apresentaram menor probabilidade de reportar doenças do aparelho osteomuscular/tecido conjuntivo $\left(\mathrm{OR}=0.60\left[\mathrm{OR}_{\mathrm{IC} 95 \%}: 0.37 ; 0.98\right]\right)$.

\section{DISCUSSÃO}

Dados do SUS (de janeiro e novembro de 2011) indicam que aproximadamente $20,5 \%$ e $3,3 \%$ de todos os 100.000 óbitos registrados foram causados por doenças do aparelho circulatório e doenças metabólicas / endócrinas / nutricionais ${ }^{19}$, respectivamente. Por outro lado, doenças do aparelho osteomuscular e do tecido conjuntivo não estão relacionados diretamente à mortalidade, mas podem comprometer a qualidade de vida da pessoa acometida e contribuir para aumentar o grau de sedentarismo e dependência por cuidados. Além disso, informações indicam que são elevados os episódios destas moléstias na população brasileira ${ }^{20}$. Embora seja necessário assumir que não se possa estabelecer uma relação de causalidade, nossos achados são relevantes ao indicar que a prática de atividades físicas está associada à menor ocorrência deste tipo de desfecho em pessoas atendidas pelo SUS e, dessa forma, possa caracteriza-se como um possível recurso não farmacológico a ser explorado.

A maioria dos pacientes $(66,7 \%)$ avaliados no presente estudo não praticava atividades físicas no lazer. Estudos semelhantes analisando usuários do SUS não 
foram encontrados. Entretanto para a população em geral e utilizando o mesmo ponto de corte para atividade física ( $\geq 180 \mathrm{~min} / \mathrm{sem})$, Fernandes et al. ${ }^{4}$ analisaram adultos de oito cidades paulista e detectaram que apenas $16,6 \%$ dos entrevistados eram fisicamente ativos. Hallal et al..$^{21}$ indicam que a prática insuficiente de atividades físicas na população adulta brasileira ultrapassa 40\% ( $<150$ minutos de atividades físicas de intensidade moderada ou vigorosa). Confrontando nossos dados com os da literatura é plausível considerar que pacientes assistidos pelo SUS apresentam taxas de sedentarismo maiores que a população em geral. Os indivíduos engajados em $\geq 180 \mathrm{~min} / \mathrm{sem}$ (que é valor de prática de atividades físicas, muito próximo da recomendação de $150 \mathrm{~min} / \mathrm{sem}$ ) foram apenas $14,7 \%$ da amostra.

Embora atividades de intensidade elevada proporcionem alto gasto energético, são mantidas por menores períodos de tempo. Assim, sabe-se que a atividade física de intensidade moderada afeta de maneira significativa o tecido adiposo, uma vez que promove lipólise para a manutenção da atividade física. Por outro lado, existe divergência na quantidade necessária de atividade física. O American College of Sports Medicine 6 através de um amplo levantamento bibliográfico concluiu que a prática de exercícios físicos de intensidade moderada/intensa por um período superior a 150 minutos de atividade por semana não é efetivo na prevenção do ganho de peso e de doenças crônicas e que, para se obter perda efetiva de peso, o ideal é que aumente o tempo da prática de exercícios físicos para pelo menos 250 minutos por semana. Nossos resultados aparentemente corroboram com tais dados. Embora os valores de atividade física “ $\geq 240$ minutos na semana" usados nesse estudo diferem da recomendação da ACSM (as questões fechadas do questionário empregado não permitiram a formação de grupos por minutos exatamente iguais ao proposto pelo ACSM: $\geq 250 \mathrm{~min} / \mathrm{sem}$ ), o engajamento em uma maior quantidade de atividade física foi associada a menor ocorrência de obesidade abdominal e as doenças do CIDM00-M99. Tais achados chamam atenção ao fato de que, 150 minutos por semana têm sido tradicionalmente indicados em campanhas de promoção a saúde, porém, aparentemente os mesmos parecem ser insuficientes e maiores quantidades sejam necessárias.

Quanto às variáveis associadas à prática suficiente de atividades físicas (>180 min./sem), observa-se que o poder aquisitivo e o sexo foram determinantes no perfil dos praticantes de exercícios. Homens e indivíduos de condição econômica mais elevada praticam mais exercícios no lazer, dados estes que corroboram com os encontrados por Fernandes et al. ${ }^{22}$. Gonçalves et al..$^{23}$ indicando que homens, quando comparados às mulheres, são estimulados pela família para a prática de atividades físicas desde a infância e este fator pode justificar os achados do estudo nesta população de adultos atendidos pelo SUS. No que se refere à condição econômica, o maior acesso a materiais esportivos e locais específicos para a sua prática pode justificar esta maior adesão durante o lazer.

Em nossa amostra, houve predominância de mulheres. Uma justificativa para esta tendência é apontada por estudos anteriores, nos quais se observa que homens evitam procurar o serviço de saúde ${ }^{24,25}$. Ainda corroborando com nossos achados, estudo realizado em Pelotas-RS identificou que a média anual de consulta das mulheres é de 4,7 , enquanto a dos homens é de $1,8^{26}$. De acordo informações prévias $^{24}$, na maior parte dos casos o motivo para a baixa procura por serviços de saúde pelos homens, está associado à masculinidade e a carga de trabalho. Estes dados ratificam a importância de se ter ajustado os modelos pelo sexo.

A principal limitação do estudo reside em seu delineamento transversal, o qual não permite o estabelecimento de relações de causalidade e, assim, estudos de de- 
lineamento prospectivos envolvendo está temática devem ser conduzidos no SUS. Por outro lado, a realização de tais estudos torna-se difícil pela falta de profissionais de Educação Física em UBS. Ainda nesse sentido, questões sobre a dificuldade de um espaço para a realização da prática de exercício poderiam ser levantadas, por isso é sempre válido lembrar a possibilidade de acordo entre as secretarias do município (saúde, ensino e esporte) para a liberação de quadras escolares e áreas de lazer (centros esportivos) para uso desses pacientes, da mesma forma existem praças e espaços abertos próximos ou mesmo dentro das UBS que poderiam ser utilizados. Vale mencionar que desde o dia 7 de abril de 2011 foi instituído o Programa Academia da Saúde no SUS, por meio da portaria n. ${ }^{\circ} 719$ do Ministério da Saúde, que tem como objetivo a implementação de polos com infraestrutura, equipamentos e quadro de pessoal qualificado para a orientação de práticas corporais e atividade física de lazer e essas academias podem ser solicitadas pela Secretária de Saúde de qualquer município.

\section{CONCLUSÃO}

O estudo apontou que, entre pacientes do SUS, é baixo o engajamento em atividades físicas no lazer, o qual foi associado à menor ocorrência de doenças do aparelho osteomuscular e do tecido conjuntivo. Além disso, sexo feminino e menor condição econômica caracterizaram-se como fatores relacionados à prática de atividades físicas no lazer.

\section{REFERÊNCIAS}

1. Ministério da Saúde. Gestão Financeira do Sistema Único de Saúde: Manual Básico. Série A. Normas e Manuais Técnicos 3a ed. revista e ampliada. 2003; Disponível em: <http://siops. datasus.gov.br/Documentacao/Manual\%20FNS.pdf> [27 março 2010].

2. Ministério da Saúde. Política Nacional de Promoção da Saúde. 2006; Disponível em: <http:// portal.saude.gov.br/portal/arquivos/pdf/PNPS2.pdf> [31 março 2011].

3. Malta CM, Castro AM, Gosch CS, Cruz DK, Bressan A, Nogueira JD, Neto OL, Temporão JG. Política Nacional da promoção de saúde e a agenda da atividade física no contexto do SUS. Rev SUS Brasil 2009;18(1):79-86.

4. Fernandes RA, Christofaro DG, Casonatto J, Codogno JS, Rodrigues EQ, Cardoso ML, Kawaguti SS, Zanesco A. Prevalence of dyslipidemia in individuals physically active during childhood, adolescence and adult age. Arq Bras Cardiol 2011;97(4):317-323.

5. Fernandes RA, Zanesco A. Early physical activity promotes lower prevalence of chronicle diseases in adulthood. Hypertens Res 2010;33:(9)926-931.

6. Donnelly JE, Blair SN, Jakicic JM, Manore MM, Rankin JW, Smith BK; American College of Sports Medicine. American College of Sports Medicine Position Stand. Appropriate physical activity intervention strategies for weight loss and prevention of weight regain for adults. Med Sci Sports Exerc 2009;41(2):459-471.

7. Sichieri R, do Nascimento S, Coutinho W. The burden of hospitalization due to overweight and obesity in Brazil. Cad Saude Publica 2007;23:(7)1721-1727.

8. Codogno JS, Fernandes RA, Sarti FM, Freitas Júnior IF, Monteiro HL. The burden of physical activity on type 2 diabetes public healthcare expenditures among adults: a retrospective study. BMC Public Health 2011;11:275.

9. Turi BC, Codogno JS, Fernandes RA, do Amaral SL, Monteiro HL. Frequência de ocorrência de doenças crônico-degenerativas em adultos com mais de 50 anos. Rev Bras Ativ Fís Saúde 2010;15(4):218-224.

10. Kilsztajn S, Silva DF, Camara MB, Ferreira VS. Grau de cobertura dos planos de saúde e distribuição regional do gasto público em saúde. Saúde Soc 2001;10(2):35-45.

11. Freitas Junior IF, Castoldi RC, Moreti DG, Pereira ML, Cardoso ML, Codogno JS, Fernandes RA, Bueno DR, Gomes JO. Aptidão física, história familiar e ocorrência de hipertensão arterial, osteoporose, doenças metabólicas e cardíacas entre mulheres. Rev SOCERJ 2009;22(3):158-164. 
12. Ministério da Saúde. Classificação Estatística Internacional de Doenças e Problemas Relacionados à Saúde. 2008; Disponível em: <http://www.datasus.gov.br/cid10/v2008/cid10. htm> [28 abril 2009].

13. Lohman TG, Roche AF, Martorell R. Anthropometric Standardization Reference Manual. Champaign: Human Kinetics Books; 1988.

14. World Health Organization. Obesity, Preventing and Managing the Global Epidemic: Report of the WHO Consultation on Obesity. 1998; Available from: <http://www.who.int/nutrition/ publications/obesity_executive_summary.pdf> [2007 Apr 13]

15. Lean ME, Han TS, Morrison CE. Waist circumference as a measure for indicating need for weight management. BMJ 1995; 311(1):158-161.

16. Baecke JA, Burema J, Frijters JE. A short questionnaire for the measurement of habitual physical activity in epidemiological studies. Am J Clin Nutr 1982;36:(6)936-942.

17. Associação Brasileira de Empresas de Pesquisa. Levantamento sócio econômico 2000-IBOPE. 2000; Disponível em: <http://www.abep.org.br> [03 jun 2008]

18. Fernandes RA, Casonatto J, Christofaro DG, Ronque ER, Oliveira AR, Freitas Júnior IF. Risk of overweight in adolescents from different socioeconomic levels. Rev Assoc Med Bras 2008;54(4):334-338.

19. Ministério da Saúde. Banco de dados do Sistema Único de Saúde. 2012; Disponível em: < www.datasus.gov.br>. [23 jan 2012].

20. Ferreira GD, Silva MC, Rombaldi AJ, Wrege ED, Siqueira FV, Hallal PC. Prevalence and associated factors of back pain in adults from southern Brazil: a population-based study. Rev Bras Fisioter 2011;15(1):31-36.

21. Hallal PC, Victora CG, Wells JC, Lima RC. Physical inactivity: prevalence and associated variables in Brazilian adults. Med Sci Sports Exerc 2003;35(11):1894-1900.

22. Fernandes RA, Christofaro DG, Casonato J, Costa Rosa CS, Costa FF, Freitas Júnior IF, Luiz Monteiro H, Ramos DE Oliveira A. Leisure time behaviors: prevalence, correlates and associations with overweight in Brazilian adults. A cross-sectional analysis. Rev Med Chil 2010;138(1):29-35.

23. Gonçalves H, Hallal PC, Amorim TC, Araújo CL, Menezes AM. Sociocultural factors and physical activity level in early adolescence. Rev Panam Salud Publica 2007;22(4):246-253.

24. Gomes R, Nascimento EF, Araújo FC. Why do men use health services less than women? Explanations by men with low versus higher education. Cad Saude Publica. 2007;23(3):565-74.

25. Figueiredo Wdos S, Schraiber LB. Male users' and primary care services health professionals' conceptions of gender and possible impacts on men's health, São Paulo, Brazil. Cien Saude Colet. 2011;16(Suppl 1):935-44.

26. da Costa JS, Reis MC, Silveira Filho CV, Linhares Rda S, Piccinini F. Prevalence of medical visits and associated factors, Pelotas, Southern Brazil, 1999-2000. Rev Saude Publica. 2008;42(6):1074-84.

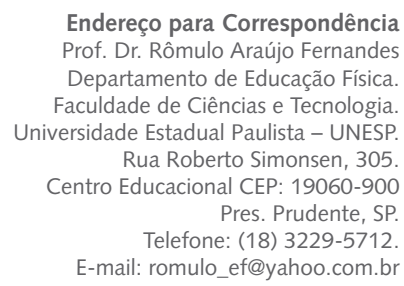

Endereço para Correspondência Prof. Dr. Rômulo Araújo Fernandes Departamento de Educação Física. Faculdade de Ciências e Tecnologia. Universidade Estadual Paulista - UNESP Rua Roberto Simonsen, 305 Centro Educacional CEP: 19060-900 Pres. Prudente, SP. Telefone: (18) 3229-5712. E-mail: romulo_ef@yahoo.com.br

Recebido 16/10/2012 Revisado 12/02/2013 Aprovado 17/02/2013 